\title{
Morita Invariance of Intrinsic Characteristic Classes of Lie Algebroids
}

\author{
Pedro FREJLICH
}

UFRGS, Departamento de Matemática Pura e Aplicada, Porto Alegre, Brasil E-mail: frejlich.math@gmail.com

Received June 18, 2018, in final form November 08, 2018; Published online November 15, 2018 https://doi.org/10.3842/SIGMA.2018.124

\begin{abstract}
In this note, we prove that intrinsic characteristic classes of Lie algebroids which in degree one recover the modular class - behave functorially with respect to arbitrary transverse maps, and in particular are weak Morita invariants. In the modular case, this result appeared in [Kosmann-Schwarzbach Y., Laurent-Gengoux C., Weinstein A., Transform. Groups 13 (2008), 727-755], and with a connectivity assumption which we here show to be unnecessary, it appeared in [Crainic M., Comment. Math. Helv. 78 (2003), 681-721] and [Ginzburg V.L., J. Symplectic Geom. 1 (2001), 121-169].
\end{abstract}

Key words: Lie algebroids; modular class; characteristic classes; Morita equivalence

2010 Mathematics Subject Classification: 53D17; 57R20

\section{Introduction}

A Lie algebroid $A$ on a manifold $M$ gives rise to intrinsic characteristic classes

$$
\operatorname{char}(A) \in \mathrm{H}^{\text {odd }}(A)
$$

in Lie algebroid cohomology, which obstruct the existence of a metric $g$ on the fibres of $\operatorname{Ad}(A):=$ $A \oplus T M$, and a connection $\nabla: T M \curvearrowright A$, whose induced basic connection $\nabla^{\text {bas }}: A \curvearrowright \operatorname{Ad}(A)$,

$$
\nabla_{a}^{\mathrm{bas}}(b, u)=\left(\nabla_{\varrho_{A} b} a+[a, b]_{A}, \varrho_{A} \nabla_{u} a+\left[\varrho_{A} a, u\right]\right), \quad a, b \in \Gamma(A), \quad u \in \mathfrak{X}(M),
$$

is $g$-metric:

$$
\mathscr{L}_{a} g\left(s, s^{\prime}\right)=g\left(\nabla_{a}^{\text {bas }} s, s^{\prime}\right)+g\left(s, \nabla_{a}^{\text {bas }} s^{\prime}\right), \quad s, s^{\prime} \in \Gamma(\operatorname{Ad}(A)) .
$$

For example, the familiar statement that there exists a Riemannian (i.e., a torsion-free and metric) connection associated with a Riemannian metric on $M$ implies that, for tangent bundles $A=T M$, these characteristic classes $\operatorname{char}(A)$ vanish.

In degree one, $\operatorname{char}^{1}(A)$ recovers the modular class of $A[10]$, the obstruction to the existence of an invariant transverse measure, first discovered in the context of Poisson manifolds [24, 31] as the 'Poisson analogue of the modular automorphism group of a von Neumann algebra'. There is an extensive literature about this important class (see the survey [21]), which is arguably the only reasonably well-understood among the intrinsic ones. It has been generalized to various geometric contexts [3, 16, 19, 20, 22, 23, 28, 29, 30], and plays a fundamental role in many constructions $[3,7,8,10,12,18,25,27,32]$.

The purpose of this short note is to show that intrinsic characteristic classes are invariant under the following version of weak Morita equivalence [13, Section 6.2]: two Lie algebroids $B$ on $N$ and $A$ on $M$ are weak Morita equivalent if there are submersions $N \stackrel{\mathbf{s}}{\leftarrow} \stackrel{\mathbf{t}}{\rightarrow} M$, and a Lie algebroid isomorphism $(\Phi, \mathrm{id}): \mathbf{s}^{!}(B) \stackrel{\sim}{\longrightarrow} \mathbf{t}^{!}(A)$ between the pullbacks of $B$ and $A$ to $\Sigma$. This establishes a correspondence between cohomology classes in $\mathrm{H}(B)$ and $\mathrm{H}(A)$, and the claim is that $\operatorname{char}(B)$ and $\operatorname{char}(A)$ are related. In fact, we prove slightly more: 
Theorem. Intrinsic characteristic classes are functorial with respect to transverse maps: if $\phi: N \rightarrow M$ is transverse to a Lie algebroid $A$ on $M$, then $\operatorname{char}\left(\phi^{!}(A)\right)=\phi^{*} \operatorname{char}(A)$.

Versions of this result have appeared in the literature in various forms; we here quote those most pertinent to our setting.

In [14, Theorem 4.2] it was shown, building on previous work [15], that the modular class is a Morita invariant for locally unimodular Poisson manifolds. Shortly afterwards, secondary and intrinsic characteristic classes were introduced (see [4, 5, 6, 11, 26]), and in [5, Corollary 8] it was proved that the intrinsic characteristic classes of Poisson manifolds of degree $(2 q-1)$ are invariant under Morita equivalences whose fibres are at least homologically $(2 q-1)$-connected; it was later extended to weak Morita equivalences of Lie algebroids under a similar connectivity condition [13, Example 6.16].

More recently, it was proved in [22, Theorem 3.10] that the modular class is functorial with respect to arbitrary transverse maps - thus dropping the connectivity condition - and the authors pose the question in [22, (iii), p. 729] about the behavior of higher intrinsic characteristic classes under morphisms. It was this question that piqued our interest, and which our Main Theorem seeks to answer.

Let us conclude these introductory remarks by pointing out that, in light of the correspondence between 2-term representations up to homotopy and VB-algebroids (see, e.g., [17]), it would be interesting to revisit the discussion below in the context of VB-algebroids and groupoids, and to explain the relationship with the results in [9] - which develops a similar line of inquiry, and through methods that bear great resemblance to the ones employed here. ${ }^{1}$

The paper is organized as follows: our conventions are discussed in Section 2, where we summarize the construction of primary, secondary and intrinsic characteristic classes of Lie algebroids from [4, 6], referring there to proofs. In Section 3 we prove our Main Theorem: as we explain there, this result is a straightforward consequence of the case of pulling back a Lie algebroid $A$ on $M$ by a submersion $p: \Sigma \rightarrow M$, and our proof, in that case, reduces to the construction of appropriate connection and metric on $\operatorname{Ad}\left(p^{!}(A)\right)$, so that the adjoint connection of $p^{!}(A)$ splits as a direct sum of the pullback of the adjoint connection of $A$ and a metric subconnection.

\section{Characteristic classes}

In this section, we give a summary of the main results and constructions needed to contextualize our discussion, referring to the appropriate references for further details.

1. For vector bundles $E$ and $D$ on $M$, we denote by $\Omega_{\mathrm{nl}}^{p}(E ; D)$ the space of nonlinear forms of degree $p$ on $E$ with values in $D$ - that is, the linear subspace of $\operatorname{Hom}\left(\wedge^{p} \Gamma(E), \Gamma(D)\right)$ consisting of those elements $\omega$ which decrease support, in the sense that $\omega\left(e_{1}, \ldots, e_{p}\right)$ is identically zero around any point around which some $e_{i} \in \Gamma(E)$ vanishes identically. When $D$ is the trivial line bundle, we write $\Omega_{\mathrm{nl}}^{p}(E)$, and we note that $\Omega_{\mathrm{nl}}^{p}(E ; D)$ is a module over $\Omega_{\mathrm{nl}}^{p}(E)$. Linear forms $\omega \in \Omega^{p}(E, D)=\Gamma\left(\wedge^{p} E^{*} \otimes D\right)$ are identified with those elements of $\Omega_{\mathrm{nl}}^{p}(E ; D)$ which are $C^{\infty}(M)$-linear in their entries. There are obvious variations when $D$ is complex or graded; see $[1,4]$.

2. Let $A$ be a Lie algebroid on $M$, and let $D$ be the graded, complex vector bundle $D_{0} \oplus D_{1}$, equipped with an odd endomorphism

$$
\partial=\left(\begin{array}{cc}
0 & \partial_{0}^{1} \\
\partial_{1}^{0} & 0
\end{array}\right): D \longrightarrow D, \quad \partial^{2}=0
$$

\footnotetext{
${ }^{1}$ I thank the anonymous referees for bringing this to my attention.
} 
A nonlinear connection of $A$ on $D$ is a linear map $\nabla: \Gamma(A) \rightarrow \operatorname{End}(\Gamma(D))$, such that, for all $a \in \Gamma(A)$,

a) $\nabla$ is a local operator;

b) $\nabla_{a}$ preserves parity;

c) $\nabla_{a}$ commutes with $\partial$;

d) $\nabla_{a}$ satisfies $\nabla_{a} f s=f \nabla_{a} s+\left(\mathscr{L}_{a} f\right) s$ for all $f \in C^{\infty}(M), s \in \Gamma(D)$.

3. A nonlinear connection of $A$ on $D$ induces:

- a derivation of degree one $\mathrm{d}_{\nabla}: \Omega_{\mathrm{nl}}(A ; D) \rightarrow \Omega_{\mathrm{nl}}(A ; D), \mathrm{d}_{\nabla} \eta\left(a_{0}, \ldots, a_{p}\right)$ being given by the usual formula

$$
\sum_{i=0}^{p}(-1)^{i} \nabla_{a_{i}} \eta\left(a_{0}, \ldots, \widehat{a_{i}}, \ldots, a_{p}\right)+\sum_{i<j}(-1)^{i+j} \eta\left(\left[a_{i}, a_{j}\right], a_{0}, \ldots, \widehat{a_{i}}, \ldots, \widehat{a_{j}}, \ldots, a_{p}\right)
$$

- a dual nonlinear connection $\nabla^{\vee}$ of $A$ on $D^{*}$, defined by the condition that

$$
\mathscr{L}_{a}\langle\theta, s\rangle=\left\langle\nabla_{a}^{\vee} \theta, s\right\rangle+\left\langle\theta, \nabla_{a} s\right\rangle, \quad a \in \Gamma(A), \quad s \in \Gamma(D), \quad \theta \in \Gamma\left(D^{*}\right),
$$

- a nonlinear connection of $A$ on $\operatorname{End}(D)$, given by $\nabla_{a} T=\left[\nabla_{a}, T\right]$, whose induced derivation $\mathrm{d}_{\nabla}: \Omega_{\mathrm{nl}}(A ; \operatorname{End}(D)) \rightarrow \Omega_{\mathrm{nl}}(A ; \operatorname{End}(D))$ is given by the graded commutator $[\nabla, \cdot]$.

4. A Hermitian metric $h$ on $D$, regarded as a complex-antilinear map $D \rightarrow D^{*}$, conjugates a nonlinear connection $\nabla$ of $A$ on $D$ to an $h$-dual nonlinear connection $\nabla^{h}$ of $A$ on $D$, given by $\nabla_{a}^{h}:=h^{-1} \circ \nabla_{a}^{\vee} \circ h$. If $\nabla=\nabla^{h}$, we say that $h$ is invariant under $\nabla$, or that $\nabla$ is $h$ metric. Note that every Hermitian metric $h$ is invariant under some nonlinear connection; e.g., $\nabla_{m}:=\frac{1}{2}\left(\nabla+\nabla^{h}\right)$.

5. A nonlinear subconnection of a nonlinear connection $\nabla$ of $A$ on $D$ is the restriction $\nabla^{\prime}=$ $\left.\nabla\right|_{D^{\prime}}$ of $\nabla$ to an invariant subbundle $D^{\prime}$, i.e., one for which $\nabla_{a} \Gamma\left(D^{\prime}\right) \subset \Gamma\left(D^{\prime}\right)$ for all $a \in \Gamma(A)$. If that is the case, there is an induced quotient nonlinear connection $\nabla / D^{\prime}$ of $A$ on $D / D^{\prime},\left(\nabla / D^{\prime}\right)_{a}[s]=\left[\nabla_{a} s\right]$. When $D=D^{\prime} \oplus D^{\prime \prime}$ where $\nabla^{\prime \prime}:=\left.\nabla\right|_{D^{\prime \prime}}$ is another subconnection, we say that $\nabla$ splits as a direct sum, and write $\nabla=\nabla^{\prime} \oplus \nabla^{\prime \prime}$.

6. For a nonlinear connection $\nabla$ of $A$ on $D, \mathrm{~d}_{\nabla}^{2}=R_{\nabla} \wedge$, where $R_{\nabla}$ denotes the curvature of $\nabla$,

$$
R_{\nabla} \in \Omega_{\mathrm{nl}}^{2}(A ; \operatorname{End}(D)), \quad R_{\nabla}(a, b)=\left[\nabla_{a}, \nabla_{b}\right]-\nabla_{[a, b]}, \quad a, b \in \Gamma(A),
$$

and it is always the case that $\mathrm{d}_{\nabla} R_{\nabla}=0$. If $R_{\nabla}=0$, we call $\nabla$ a nonlinear representation. Because the supertrace $\operatorname{Tr}_{s}(T)=\operatorname{Tr}\left(T_{00}\right)-\operatorname{Tr}\left(T_{11}\right)$ induces a linear map intertwining derivations,

$$
\operatorname{Tr}_{s}: \Omega_{\mathrm{nl}}(A ; \operatorname{End}(D)) \longrightarrow \Omega_{\mathrm{nl}}(A ; \mathbb{C}), \quad \mathrm{d}_{A} \operatorname{Tr}_{s}=\operatorname{Tr}_{s} \mathrm{~d}_{\nabla}
$$

it follows in general that $\operatorname{Tr}_{s}\left(R_{\nabla}^{q}\right) \in \Omega_{\mathrm{nl}}^{2 q}(A ; \mathbb{C})$ are $\mathrm{d}_{A}$-closed for every integer $q$; see [4].

7. If $(\Phi, \phi): B \rightarrow A$ is a morphism of Lie algebroids, and $\nabla$ is a nonlinear connection of $A$ on $D$, there is an induced pullback nonlinear connection $(\Phi, \phi)^{!} \nabla$ of $B$ on $\phi^{*}(D)$,

$$
(\Phi, \phi)^{!} \nabla_{a} \phi^{\dagger}(s):=\phi^{\dagger}\left(\nabla_{\Phi(a)} s\right), \quad a \in \Gamma\left(\phi^{!}(A)\right), \quad s \in \Gamma(D),
$$


in which case $(\Phi, \phi):(\Phi, \phi)^{!} \nabla \rightarrow \nabla$ defines a pullback morphism of nonlinear connections, in the sense that the induced linear map

$$
(\Phi, \phi)^{*}: \Omega_{\mathrm{nl}}(A ; D) \rightarrow \Omega_{\mathrm{nl}}\left(B ; \phi^{*}(D)\right)
$$

intertwines the derivations $\mathrm{d}_{\nabla}$ and $\mathrm{d}_{(\Phi, \phi)} ! \nabla \cdot{ }^{2}$ If $\phi: N \rightarrow M$ is a smooth map transverse to a Lie algebroid $A$ on $M$, i.e.,

$$
\phi_{*}\left(T_{x} N\right)+\varrho_{A}\left(A_{\phi(x)}\right)=T_{\phi(x)} M, \quad x \in N,
$$

then there is a pullback Lie algebroid $\phi^{!}(A):=T N \times_{T M} A$ on $N$, and an induced pullback morphism of Lie algebroids $(\widetilde{\phi}, \phi): \phi^{!}(A) \rightarrow A$. In this case, we will write simply $\phi^{!} \nabla$ and $\phi^{*}$ instead of $(\widetilde{\phi}, \phi)^{!} \nabla$ and $(\widetilde{\phi}, \phi)^{*}$.

8. A nonlinear connection is a connection tout court if

$$
\nabla_{f a} s=f \nabla_{a} s, \quad f \in C^{\infty}(M), \quad a \in \Gamma(A), \quad s \in \Gamma(D),
$$

that is, if it is $C^{\infty}(M)$-linear in the $\Gamma(A)$-entry, in which case we write $\nabla: A \curvearrowright D$. Two nonlinear connections $\nabla^{0}, \nabla^{1}$ are equivalent provided that there exists $\theta \in \Omega_{\mathrm{nl}}^{1}(A ; \operatorname{End}(D))$, such that

$$
\nabla_{a}^{1}-\nabla_{a}^{0}=[\theta(a), \partial], \quad a \in \Gamma(A),
$$

in which case $\operatorname{Tr}_{s}\left(R_{\nabla_{0}}^{q}\right)=\operatorname{Tr}_{s}\left(R_{\nabla_{1}}^{q}\right)$ for all $q$ (see [6]). A nonlinear connection $\nabla$ of $A$ on $D$ will be called a connection up to homotopy if it is equivalent to a connection; in this case, we will write $\nabla: A \cup D$. Both connections and connections up to homotopy are preserved by all operations on nonlinear connections described in items 3-7. Note that, for a connection up to homotopy $\nabla, \operatorname{Tr}_{s}\left(R_{\nabla}^{q}\right)$ are linear forms, $\operatorname{Tr}_{s}\left(R_{\nabla}^{q}\right) \in \Omega^{2 q}(A)$. A representation up to homotopy ${ }^{3}$ is a connection up to homotopy for which $R_{\nabla}$ vanishes identically, in which case $\mathrm{d}_{\nabla}$ turns $\Omega_{\mathrm{nl}}^{\bullet}(A ; D)$ into a cochain complex.

In the remainder of this section, we recall the discussion in [6], referring there to proofs and further details.

Lemma. There is a rule cs which assigns to all non-negative integers $p, q \geqslant 0$ and connections up to homotopy $\nabla_{0}, \ldots, \nabla_{p}: A \cup D$, a cochain

$$
\operatorname{cs}^{q}\left(\nabla_{0}, \ldots, \nabla_{p}\right) \in \Omega^{2 q-p}(A ; \mathbb{C})
$$

with the property that, for every permutation $\sigma$ and Hermitian metric $h$ on $D$ :

CS1) $\operatorname{cs}^{q}(\nabla)=\operatorname{Tr}_{s}\left(R_{\nabla}^{q}\right)$,

CS2) $\operatorname{cs}^{q}\left(\nabla_{\sigma(0)}, \ldots, \nabla_{\sigma(p)}\right)=(-1)^{\sigma} \operatorname{cs}^{q}\left(\nabla_{0}, \ldots, \nabla_{p}\right)$,

CS3) $\mathrm{d}_{A} \operatorname{cs}^{q}\left(\nabla_{0}, \ldots, \nabla_{p}\right)=\sum_{i=0}^{p}(-1)^{i} \operatorname{cs}^{q}\left(\nabla_{0}, \ldots, \widehat{\nabla}_{i}, \ldots, \nabla_{p}\right)$,

CS4) $\operatorname{cs}^{q}\left(\nabla_{0}^{h}, \ldots, \nabla_{p}^{h}\right)=(-1)^{q} \overline{\operatorname{cs}^{q}\left(\nabla_{0}, \ldots, \nabla_{p}\right)}$.

\footnotetext{
${ }^{2}$ As explained in [22], it is best to think that a connection $\nabla: A \curvearrowright D$ induces the derivation $\mathrm{d}_{\nabla \vee}$, simply because the map of modules induced by a pair of vector bundle maps $\Phi: B \rightarrow A$ and $\Psi: D_{B} \rightarrow D_{A}$ covering the same smooth map $\phi: N \rightarrow M$ is $(\Phi, \Psi, \phi)^{*}: \Omega\left(A ; D_{A}^{*}\right) \rightarrow \Omega\left(B ; D_{B}^{*}\right)$. A morphism from a connection $\nabla_{B}: B \curvearrowright D_{B}$ to a connection $\nabla_{A}: A \curvearrowright D_{A}$ is then a such triple $(\Phi, \Psi, \phi)$ for which $(\Phi, \Psi, \phi)^{*}$ intertwines the derivations $\mathrm{d}_{\nabla}{ }_{A}$ and $\mathrm{d}_{\nabla_{B}^{\vee}}$. When $\Psi$ is fibrewise an isomorphism - as in the case of a pullback morphism - we may dualize the construction above to a map of modules $\Omega\left(A ; D_{A}\right) \rightarrow \Omega\left(B ; D_{B}\right)$ intertwining the derivations $\mathrm{d}_{\nabla_{A}}$ and $\mathrm{d}_{\nabla_{B}}$.

${ }^{3}$ For the convenience of the reader, we chose to maintain the term representation up to homotopy as it appears in $[4,6]$, in spite of the fact that terminology has come to mean something else [1].
} 
Such cochains are given explicitly by

$$
\operatorname{cs}^{q}\left(\nabla_{0}, \ldots, \nabla_{p}\right):= \begin{cases}\operatorname{Tr}_{s}\left(R_{\nabla_{0}}^{q}\right) & \text { if } p=0, \\ (-1)^{\left\lfloor\frac{p+1}{2}\right\rfloor} f_{\Delta^{p}} \operatorname{Tr}_{s}\left(R_{\nabla^{\text {aff }}}^{q}\right) & \text { if } p>0,\end{cases}
$$

where $\lfloor t\rfloor$ the greatest integer no greater than $t$ and:

- $f_{\Delta^{p}}: \Omega^{\bullet}\left(\operatorname{pr}^{!}(A) ; \mathbb{C}\right) \rightarrow \Omega^{\bullet-p}(A ; \mathbb{C})$ denotes the linear map of fibre integration ${ }^{4}$ associated to the canonical projection from the product of $M$ with the standard $p$-simplex pr: $M \times$ $\Delta^{p} \rightarrow M$

- $\nabla^{\mathrm{aff}}: \operatorname{pr}^{!}(A) \cup \operatorname{pr}^{*}(D)$ denotes the connection up to homotopy $\nabla^{\mathrm{aff}}=\sum_{i=0}^{p} t_{i} \operatorname{pr}^{!}\left(\nabla_{i}\right)$.

Given a connection up to homotopy $\nabla: A \cup D$, define

$$
\operatorname{cs}(\nabla)=\operatorname{Tr}_{s}\left(\exp \left(i R_{\nabla}\right)\right)=\sum \frac{i^{q}}{q !} \operatorname{cs}^{q}(\nabla) \in \Omega(A ; \mathbb{C}) .
$$

Proposition 1 (primary characteristic classes).

a) For a connection up to homotopy, we have $\mathrm{d}_{A} \operatorname{cs}(\nabla)=0$ and $\operatorname{cs}\left(\nabla_{0} \oplus \nabla_{1}\right)=\operatorname{cs}\left(\nabla_{0}\right)+\operatorname{cs}\left(\nabla_{1}\right)$;

b) for all Lie algebroid morphisms $(\Phi, \phi): B \rightarrow A$ and connection up to homotopy $\nabla: A \cup D$, we have $\operatorname{cs}\left((\Phi, \phi)^{!}(\nabla)\right)=(\Phi, \phi)^{*} \operatorname{cs}(\nabla)$;

c) the cohomology class $[\operatorname{cs}(\nabla)]$ does not depend on the choice of connection up to homotopy $\nabla$;

d) $[\operatorname{cs}(\nabla)] \in \mathrm{H}^{2 \bullet}(A)$ is a real cohomology class lying in the image of the map $\left(\varrho_{A}, \mathrm{id}\right)^{*}: \mathrm{H}(M) \rightarrow$ $\mathrm{H}(A)$ induced by the anchor of $A$;

e) $[\operatorname{cs}(\nabla)] \in \mathrm{H}^{4 \bullet}(A)$ if $\nabla$ is a real ${ }^{5}$ connection up to homotopy.

We call the Chern character of $D$ the element $\operatorname{ch}(D) \in \mathrm{H}(A)$ represented by $\operatorname{cs}(\nabla)$, for some connection up to homotopy $\nabla: A \cup D$. We regard it as a primary characteristic class, obstructing the existence of a representation up to homotopy of $A$ on $D$. The vanishing of $\operatorname{cs}(\nabla)$ allows one to define secondary characteristic classes $\mathfrak{u}(\nabla) \in \mathrm{H}^{\mathrm{odd}}(A)$, which obstruct the existence of an invariant metric. For a connection up to homotopy $\nabla: A \cup D$ and a Hermitian metric $h$ on $D$, define

$$
\mathrm{u}(\nabla, h):=\sum i^{q+1} \operatorname{cs}^{q}\left(\nabla, \nabla^{h}\right) \in \Omega^{\text {odd }}(A ; \mathbb{C}) .
$$

Proposition 2 (secondary characteristic classes).

a) The cochains $\mathrm{u}(\nabla, h)$ are real;

b) for all Lie algebroid morphism $(\Phi, \phi): B \rightarrow A$, connection up to homotopy $\nabla: A \cup D$ and Hermitian metric $h$, we have $\mathrm{u}\left((\Phi, \phi)^{!}(\nabla), \phi^{*}(h)\right)=(\Phi, \phi)^{*} \mathrm{u}(\nabla, h)$;

\footnotetext{
${ }^{4}$ To construct $f_{\Delta^{p}}$, fix a splitting $\sigma: \operatorname{pr}^{*}(A) \rightarrow \mathrm{pr}^{!}(A)$ to $\widetilde{\mathrm{pr}}$, and denote by q: $\Omega\left(\mathrm{pr}^{!} A\right) \rightarrow \Omega(V)$ the homomorphism induced by the inclusion of $V=\operatorname{ker}_{*} \subset T\left(M \times \Delta^{p}\right)$. Then for $\omega \in \Omega^{p+q}\left(\operatorname{pr}^{!}(A)\right)$ and sections $a_{1}, \ldots, a_{q} \in \Gamma(A)$, define $f_{\Delta^{p}} \omega$ so that the identity below is satisfied:

$$
\iota_{a_{q}} \cdots \iota_{a_{1}} f_{\Delta^{p}} \omega:=\int_{\Delta^{p}} \mathrm{q}\left(\iota_{\sigma\left(a_{q}\right)} \cdots \iota_{\sigma\left(a_{1}\right)} \omega\right) .
$$

${ }^{5}$ We consider real vector bundles $D$ as complex ones via complexification $D \otimes_{\mathbb{R}} \mathbb{C}$, and we observe that a real nonlinear connection $\nabla$ of $A$ on $D$ induces a complex nonlinear connection $\nabla_{\mathbb{C}}$ of $A$ on $D \otimes_{\mathbb{R}} \mathbb{C}$, and that a metric $g$ on $D$ induces a Hermitian metric $g_{\mathbb{C}}$ on the complexification $D \otimes_{\mathbb{R}} \mathbb{C}$, in such a way that $\left(\nabla^{g}\right)_{\mathbb{C}}=\left(\nabla_{\mathbb{C}}\right)^{g_{\mathbb{C}}}$.
} 
c) If $\operatorname{cs}(\nabla)=0$, then $\mathrm{d}_{A} \mathrm{u}(\nabla, h)=0$, in which case:

i) $\mathfrak{u}(\nabla):=[\mathrm{u}(\nabla, h)] \in \mathrm{H}^{\text {odd }}(A)$ is independent of $h$;

ii) $\mathfrak{u}(\nabla) \in \mathrm{H}^{4 \bullet+1}(A)$ if $\nabla$ is a real connection up to homotopy.

Main Example. Let the adjoint bundle $\operatorname{Ad}(A)$ of a Lie algebroid $A$ on $M$ be $A$ in even parity, and $T M$ in odd parity, equipped with $\partial(a, u)=\left(0, \varrho_{A}(a)\right)$. Then $\nabla^{\text {ad }}$ given by

$$
\nabla_{a}^{\mathrm{ad}}(b, u):=\left([a, b]_{A},\left[\varrho_{A} a, u\right]\right)
$$

defines a representation up to homotopy. This can be seen as follows: every linear connection $\nabla: T M \curvearrowright A$ induces a linear basic connection $\nabla^{\text {bas }}: A \curvearrowright \operatorname{Ad}(A)$,

$$
\nabla_{a}^{\mathrm{bas}}(b, u):=\left(\nabla_{\varrho_{A} b} a+[a, b]_{A}, \varrho_{A} \nabla_{u} a+\left[\varrho_{A} a, u\right]\right) ;
$$

and the nonlinear representation $\nabla^{\text {ad }}$ is equivalent to $\nabla^{\text {bas }}$

$$
\nabla^{\mathrm{ad}}=\nabla^{\mathrm{bas}}+\left[\theta_{\nabla}, \partial\right], \quad \theta_{\nabla}(a)(b, u):=\left(\nabla_{u} a, 0\right) .
$$

$\nabla^{\text {ad }}$ can be alternatively defined as the unique representation up to homotopy which under the canonical Lie algebroid map (pr,id) $: J_{1}(A) \rightarrow A$ pulls back to the canonical representation

$$
\nabla^{j_{1}}: J_{1}(A) \curvearrowright \operatorname{Ad}(A), \quad \nabla_{j_{1} a}^{j_{1}}(b, u)=\nabla_{a}^{\mathrm{ad}}(b, u) .
$$

Definition. The intrinsic characteristic classes $\operatorname{char}^{q}(A) \in \mathrm{H}^{2 q-1}(A)$ of the Lie algebroid $A$ are the secondary characteristic classes $\mathfrak{u}^{q}\left(\nabla^{\text {ad }}\right)$ of the adjoint representation up to homotopy $\nabla^{\text {ad }}$.

Note that it follows from the discussion in the Main Example, and item b) of Proposition 2, that $\operatorname{char}(A)$ can be alternatively defined as the unique element $\mathrm{H}^{\text {odd }}(A)$ which pulls back under the Lie algebroid map (pr,id): $J_{1}(A) \rightarrow A$ to the secondary characteristic class $\mathfrak{u}\left(\nabla^{j_{1}}\right)$ of the canonical representation of $J_{1}(A)$ on $\operatorname{Ad}(A)$.

Example. The modular class $\bmod (A)$ of $A$ coincides with $2 \pi \operatorname{char}^{1}(A) \in \mathrm{H}^{1}(A)$.

Remark. Intrinsic characteristic classes are not a complete obstruction to the existence of a metric which is invariant under a basic connection. This is in contrast to the case of the modular class, whose vanishing implies the existence of an invariant measure. As an example, let $\mathfrak{g}$ be the 3-dimensional Lie algebra given by

$$
\left[e_{1}, e_{2}\right]=0, \quad\left[e_{1}, e_{3}\right]=a e_{1}+b e_{2}, \quad\left[e_{2}, e_{3}\right]=c e_{1}+d e_{2}, \quad Q=\left(\begin{array}{cc}
a & b \\
c & d
\end{array}\right) \in \mathrm{GL}_{2}(\mathbb{R}),
$$

which we regard as a Lie algebroid over a point. By dimensional reasons, we have

$$
\operatorname{char}(\mathfrak{g})=0 \quad \Longleftrightarrow \bmod (\mathfrak{g})=0 \quad \Longleftrightarrow \quad \operatorname{Tr} Q=0 .
$$

On the other hand, the only basic connection is $\nabla_{x}^{\text {bas }} y=[x, y]$, and $\operatorname{Ad}(\mathfrak{g})$ admits a (positivedefinite) ad-invariant metric iff $\mathfrak{g}$ is abelian.

\section{Proof of the Main Theorem}

While primary and secondary characteristic classes are functorial with respect to pullbacks essentially by inspection of the construction, for intrinsic characteristic classes the situation is slightly more intricate because the adjoint representation up to homotopy of a pullback is not itself a pullback representation up to homotopy. The following special case will turn out to be key: 
Proposition 3. Intrinsic characteristic classes are functorial with respect to surjective submersions.

The proof of the Main Theorem requires the following direct consequence of Proposition 3:

Proposition 4. Intrinsic characteristic classes are functorial with respect to transverse, closed embeddings.

Proof. Let $i: X \hookrightarrow M$ be a closed embedding transverse to $A$, and $p: N X:=\left.T M\right|_{X} / T X \rightarrow X$ the normal bundle to $X$. By the normal form theorem in [2], we can find an open subset $U \subset N X$, and an isomorphism of Lie algebroids $(\Phi, \phi):\left.p^{!} \imath^{!}(A) \stackrel{\sim}{\longrightarrow} A\right|_{\phi(U)}$, such that the following triangle of morphisms of Lie algebroids commutes

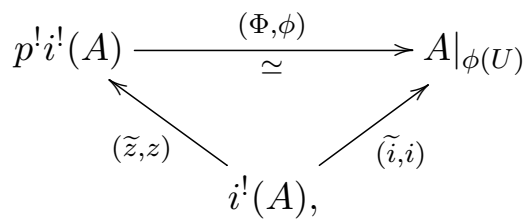

where $(\widetilde{i}, i)$ is the pullback morphism of Lie algebroids induced by the inclusion $i: X \hookrightarrow M$, and $(\widetilde{z}, z)$ is the pullback morphism of Lie induced by the zero section $z: X \hookrightarrow U$. Because $(\Phi, \phi)$ is an isomorphism, we have that char $\left(p^{!} i^{!}(A)\right)=(\Phi, \phi)^{*} \operatorname{char}(A)$, and this implies

$$
i^{*} \operatorname{char}(A)=z^{*} \operatorname{char}\left(p^{!} i^{!}(A)\right)=(p z)^{*} \operatorname{char}\left(i^{!}(A)\right)=\operatorname{char}\left(i^{!}(A)\right),
$$

where in the middle equality we used Proposition 3.

Proof of the Main Theorem. Let $\phi: N \rightarrow M$ be a smooth map, and $A$ a Lie algebroid on $M$. Factor $\phi$ as $\operatorname{pr}_{2} i$, where

$$
N \stackrel{\mathrm{pr}_{1}}{\longleftarrow} N \times M \stackrel{\mathrm{pr}_{2}}{\longrightarrow} M
$$

denote the canonical projections, and where

$$
i: N \longrightarrow N \times M, \quad i(x):=(x, \phi(x))
$$

is the embedding of $N$ as the graph of $\phi$. Because $\operatorname{pr}_{2}$ is a surjective submersion, $\phi$ is transverse to $A$ exactly when $i$ is transverse to $\mathrm{pr}_{2}^{!}(A)$. Hence

$$
\phi^{*} \operatorname{char}(A)=i^{*} \operatorname{pr}_{2}^{*} \operatorname{char}(A)=i^{*} \operatorname{char}\left(\operatorname{pr}_{2}^{!} A\right)=\operatorname{char}\left(i^{!} \operatorname{pr}_{2}^{!} A\right)=\operatorname{char}\left(\phi^{!} A\right),
$$

where in the second equality we used Proposition 3, and in the third, Proposition 4.

So everything boils down to

Proof of Proposition 3. Let $p: \Sigma \rightarrow M$ be a surjective submersion, and $A$ a Lie algebroid on $M$. Our goal is to show that

$$
\operatorname{char}\left(p^{!}(A)\right)=p^{*}(\operatorname{char}(A)),
$$

and by item b) of Proposition 2, it suffices to show that

$$
\operatorname{char}\left(p^{!}(A)\right)=\mathfrak{u}\left(p^{!}\left(\nabla^{\text {bas }}\right)\right) \text {, }
$$

where $\nabla^{\text {bas }}$ is the basic connection associated (in the sense of the Main Example) with some linear connection $\nabla: T M \curvearrowright A$. 
To do so, it is enough to give a recipe which to a connection $\nabla: T M \curvearrowright A$ and metrics $g_{A}$ on $A$ and $g_{M}$ on $T M$, assigns a connection $\bar{\nabla}: T \Sigma \curvearrowright p^{!}(A)$, and metrics $g_{p^{!}(A)}$ on $p^{!}(A)$ and $g_{\Sigma}$ on $T \Sigma$, such that

$$
\operatorname{cs}\left(\bar{\nabla}^{\text {bas }}, \bar{\nabla}^{\text {bas }, \bar{g}}\right)=p^{*} \operatorname{cs}\left(\nabla^{\text {bas }}, \nabla^{\text {bas }, g}\right)
$$

where $g=\left(g_{A}, g_{M}\right)$ and $\bar{g}=\left(g_{p^{!}(A)}, g_{\Sigma}\right)$. Our recipe for $\left(\bar{\nabla}, g_{p^{!}(A)}, g_{\Sigma}\right)$ will depend on choices of a metric $g_{V}$ on the vertical bundle $V=\operatorname{ker} p_{*}$, and an Ehresmann connection $H \subset T \Sigma$ for $p$, all of which we fix once and for all. Denote by $h: p^{*}(T M) \rightarrow T \Sigma$ the horizontal lift associated with $H$ and by $\mathbb{V}$ the subbundle $V \oplus V \subset \operatorname{Ad}\left(p^{!}(A)\right)$.

Consider the exact sequence of vector bundles over $\Sigma$ :

$$
0 \longrightarrow \mathbb{V} \longrightarrow \operatorname{Ad}\left(p^{!}(A)\right) \stackrel{\widetilde{p}}{\longrightarrow} p^{*} \operatorname{Ad}(A) \longrightarrow 0
$$

and define

$$
\text { hor: } p^{*}(A) \stackrel{\sim}{\longrightarrow} C \subset p^{!}(A), \quad \operatorname{hor}(a):=\left(h\left(\varrho_{A} a\right), a\right) \in T \Sigma \times_{T M} A .
$$

This induces a linear splitting (hor, $h): p^{*} \operatorname{Ad}(A) \rightarrow \operatorname{Ad}\left(p^{!}(A)\right)$ to the exact sequence above, and we define metrics $g_{\Sigma}$ on $T \Sigma$ and $g_{p ! A}$ on $p^{!}(A)$ so that

$$
\begin{array}{lll}
\left(V, g_{V}\right) \oplus\left(p^{*}(T M), p^{*} g_{M}\right) \longrightarrow\left(T \Sigma, g_{\Sigma}\right), & \left(v, p^{\dagger}(u)\right) & \mapsto v+h(u), \\
\left(V, g_{V}\right) \oplus\left(p^{*}(A), p^{*} g_{A}\right) \longrightarrow\left(p^{!} A, g_{p^{\prime} A}\right), & \left(v, p^{\dagger}(a)\right) \mapsto v+\operatorname{hor}(a)
\end{array}
$$

be isometries.

The metric $\bar{g}=\left(g_{p^{!}(A)}, g_{\Sigma}\right)$ on $\operatorname{Ad}\left(p^{!}(A)\right)$ is the one in the output of our recipe. The construction of $\bar{\nabla}$ which satisfies (3.1), on the other hand, is subtler, and proceeds in steps.

Step one. First consider the Riemannian connection $\nabla^{\mathrm{R}}: T \Sigma \curvearrowright T \Sigma$ of $g_{\Sigma}$, which satisfies

$$
\nabla^{\mathrm{R}, \text { bas }}=\nabla^{\mathrm{R}}=\nabla^{\mathrm{R}, g_{\Sigma}} .
$$

Step two. Let the horizontal and vertical projections corresponding to $g_{\Sigma}$ be denoted by $\mathrm{P}_{H}, \mathrm{P}_{V}: T \Sigma \rightarrow T \Sigma$, and define a new connection

$$
\nabla^{\Sigma}: T \Sigma \curvearrowright T \Sigma, \quad \nabla_{u}^{\Sigma} v:=\mathrm{P}_{H} \nabla_{u}^{\mathrm{R}} \mathrm{P}_{H}(v)+\mathrm{P}_{V} \nabla_{u}^{\mathrm{R}} \mathrm{P}_{V}(v) .
$$

Note that $V, H \subset T \Sigma$ are subconnections by construction. We claim that $\nabla^{\Sigma}$ is $g_{\Sigma}$-metric, $\nabla^{\Sigma}=\nabla^{\Sigma, g_{\Sigma}}$. Indeed, note that by definition of $\nabla^{\Sigma}$, we have

$$
g_{\Sigma}\left(\nabla_{u}^{\Sigma} v, w\right)=g_{\Sigma}\left(\nabla_{u}^{\mathrm{R}} \mathrm{P}_{H} v, \mathrm{P}_{H} w\right)+g_{\Sigma}\left(\nabla_{u}^{\mathrm{R}} \mathrm{P}_{V} v, \mathrm{P}_{V} w\right)
$$

and because $g_{\Sigma}(V, H)=0$ and $\nabla^{\mathrm{R}}$ is $g_{\Sigma^{-}}$metric,

$$
g_{\Sigma}\left(\nabla_{u}^{\Sigma} v, w\right)+g_{\Sigma}\left(v, \nabla_{u}^{\Sigma} w\right)=\mathscr{L}_{u} g_{\Sigma}\left(\mathrm{P}_{H} v, \mathrm{P}_{H} w\right)+\mathscr{L}_{u} g_{\Sigma}\left(\mathrm{P}_{V} v, \mathrm{P}_{V} w\right)=\mathscr{L}_{u} g_{\Sigma}(v, w) .
$$

Step three. There exist unique $C^{\infty}(\Sigma)$-linear maps

$$
\mathcal{D}: \quad \Gamma(H) \rightarrow \operatorname{End}(\Gamma(V)), \quad \mathcal{E}: \quad \Gamma(H) \longrightarrow \operatorname{End}(\Gamma(C)),
$$

satisfying the Leibniz rule

$$
\mathcal{D}_{w}(f v)=f \mathcal{D}_{w}(v)+\left(\mathscr{L}_{w} f\right) v, \quad \mathcal{E}_{w}(f \alpha)=f \mathcal{E}_{w}(\alpha)+\left(\mathscr{L}_{w} f\right) \alpha
$$


for all $f \in C^{\infty}(\Sigma), v \in \Gamma(V), w \in \Gamma(H)$ and $\alpha \in \Gamma(C)$, and such that

$$
\mathcal{D}_{h(u)} v=[h(u), v], \quad \mathcal{E}_{h(u)} \operatorname{hor}(a)=\operatorname{hor}\left(\nabla_{u} a\right),
$$

for all $u \in \mathfrak{X}(M)$ and $a \in \Gamma(A)$. Concretely, we identify $\Gamma(H)$ with $C^{\infty}(\Sigma) \otimes_{C^{\infty}(M)} \mathfrak{X}(M)$ and $\Gamma(C)$ with $C^{\infty}(\Sigma) \otimes_{C^{\infty}(M)} \Gamma(A)$. Then $\mathcal{D}$ is just obtained by extension of scalars $\mathcal{D}_{\lambda h(u)}:=$ $\lambda \mathcal{D}_{h(u)}$. In turn, for each fixed $u \in \mathfrak{X}(M)$, the linear map

$$
\mathcal{E}_{h(u)}: \Gamma(A) \longrightarrow \Gamma(C), \quad \mathcal{E}_{h(u)}(a)=\operatorname{hor}\left(\nabla_{u} a\right)
$$

extends to an endomorphism of $\Gamma(C)$ via $\mathcal{E}_{h(u)}(\mu \otimes a):=\mu \mathcal{E}_{h(u)}(a)+\left(\mathscr{L}_{h(u)} \mu\right) \operatorname{hor}(a)$, and $\mathcal{E}$ is obtained by extension of scalars: $\mathcal{E}_{\lambda h(u)}:=\lambda \mathcal{E}_{h(u)}$.

Step four. Let now $\bar{\nabla}: T \Sigma \curvearrowright p^{!}(A)$ be the connection which satisfies
a) $\bar{\nabla}_{v} v^{\prime}:=\nabla_{v}^{\Sigma, \text { bas }} v^{\prime}$
b) $\bar{\nabla}_{w} v^{\prime}:=\mathcal{D}_{w}\left(v^{\prime}\right)$,
c) $\bar{\nabla}_{v} \alpha:=\nabla_{v}^{\Sigma, \text { bas }} \varrho_{p^{!}(A)} \alpha$,
d) $\bar{\nabla}_{w} \alpha:=\mathcal{E}_{w} \alpha+c\left(w, \varrho_{p^{!}(A)} \alpha\right)$

for all $v, v^{\prime} \in \Gamma(V), w \in \Gamma(H)$ and $\alpha \in \Gamma(C)$, and where $c$ denotes the extension of

$$
\mathfrak{X}(M) \times \mathfrak{X}(M) \longrightarrow \Gamma(V), \quad\left(u, u^{\prime}\right) \mapsto\left[h(u), h\left(u^{\prime}\right)\right]-h\left[u, u^{\prime}\right]
$$

to a form $c \in \Gamma\left(\wedge^{2} p^{*}\left(T^{*} M\right) \otimes V\right)$. This concludes our recipe

$$
\left(\nabla, g_{A}, g_{M}\right) \mapsto\left(\bar{\nabla}, g_{p^{!}(A)}, g_{\Sigma}\right)
$$

and all there is left to do is to check that (3.1) is satisfied.

We begin by computing the basic connection $\bar{\nabla}^{\text {bas }}: p^{!}(A) \curvearrowright \operatorname{Ad}\left(p^{!}(A)\right)$ :
a) $\bar{\nabla}_{v}^{\text {bas }} v^{\prime}=\nabla_{v}^{\Sigma} v^{\prime}$
b) $\bar{\nabla}_{\mathrm{hor}(a)}^{\mathrm{bas}} v^{\prime}=\nabla_{h\left(\varrho_{A} a\right)}^{\Sigma} v^{\prime}$,
c) $\bar{\nabla}_{v}^{\text {bas }} \operatorname{hor}(b)=0$,
d) $\bar{\nabla}_{v}^{\text {bas }} h(u)=0$,
e) $\bar{\nabla}_{\mathrm{hor}(a)}^{\text {bas }} \operatorname{hor}(b)=\operatorname{hor}\left(\nabla_{a}^{\text {bas }} b\right)$,
f) $\bar{\nabla}_{\mathrm{hor}(a)}^{\mathrm{bas}} h(u)=h\left(\nabla_{a}^{\mathrm{bas}} u\right)$,

where $v, v^{\prime} \in \Gamma(V), u \in \mathfrak{X}(M)$ and $a, b \in \Gamma(A)$. In particular, it follows from a) and b) that

$$
\bar{\nabla}_{\alpha}^{\text {bas }} \Gamma(\mathbb{V}) \subset \Gamma(\mathbb{V}), \quad \alpha \in \Gamma\left(p^{!}(A)\right),
$$

whereas from c)-f) it follows that

$$
\bar{\nabla}_{\alpha}^{\text {bas }} \Gamma\left(p^{*} \operatorname{Ad}(A)\right) \subset \Gamma\left(p^{*} \operatorname{Ad}(A)\right), \quad \alpha \in \Gamma\left(p^{!}(A)\right) .
$$

Because $\mathbb{V}$ and $p^{*} \operatorname{Ad}(A)$ are $\bar{g}$-orthogonal, it follows from (3.2), (3.3) and the definition of $\bar{g}$-dual connection that

$$
\bar{\nabla}_{\alpha}^{\text {bas }, \bar{g}} \Gamma(\mathbb{V}) \subset \Gamma(\mathbb{V}), \quad \bar{\nabla}_{\alpha}^{\text {bas }, \bar{g}} \Gamma\left(p^{*} \operatorname{Ad}(A)\right) \subset \Gamma\left(p^{*} \operatorname{Ad}(A)\right), \quad \alpha \in \Gamma\left(p^{!}(A)\right) .
$$

The explicit description a)-f) of $\bar{\nabla}^{\text {bas }}$ also implies that $\bar{\nabla}^{\text {bas }}$ restricts to a subconnection $\nabla^{\mathbb{V}}:=$ $\left.\bar{\nabla}^{\text {bas }}\right|_{\mathbb{V}}: p^{!}(A) \curvearrowright \mathbb{V}$, which is $\bar{g}$-metric:

$$
\nabla^{\mathbb{V}}=\left.\bar{\nabla}^{\text {bas }}\right|_{\mathbb{V}}=\left.\bar{\nabla}^{\text {bas }, \bar{g}}\right|_{\mathbb{V}}
$$

and that for $\alpha, \beta \in \Gamma\left(p^{!}(A)\right), a, b \in \Gamma(A), w \in \mathfrak{X}(\Sigma)$ and $u \in \mathfrak{X}(M)$,

$$
\alpha \sim_{p} a, \quad \beta \sim_{p} b, \quad w \sim_{p} u \quad \Longrightarrow \quad \bar{\nabla}_{\alpha}^{\text {bas }}(\beta, w) \sim_{p} \nabla_{a}^{\text {bas }}(b, u) .
$$


We conclude from equations (3.2), (3.3), (3.5) and (3.6) that

$$
\bar{\nabla}^{\text {bas }}=\nabla^{\mathbb{V}} \oplus p^{!}\left(\nabla^{\text {bas }}\right) \text {. }
$$

Because $p^{*} g\left(b, b^{\prime}\right)=\bar{g}\left(\operatorname{hor}(b), \operatorname{hor}\left(b^{\prime}\right)\right)$ and $\bar{\nabla}_{v}^{\text {bas }}$ hor $(b)=0$, it follows that

$$
\bar{\nabla}_{v}^{\text {bas }, \bar{g}} \operatorname{hor}(b)=0, \quad v \in \Gamma(V), \quad b \in \Gamma(A),
$$

and because $\bar{\nabla}_{\text {hor }(a)}^{\text {bas }} \operatorname{hor}(b)=\operatorname{hor}\left(\nabla_{a}^{\text {bas }} b\right)$, it follows that

$$
\bar{\nabla}_{\text {hor }(a)}^{\text {bas }, \bar{g}} \operatorname{hor}(b)=\operatorname{hor}\left(\nabla_{a}^{\text {bas }, g} b\right),
$$

whence

$$
\alpha \sim_{p} a, \quad \beta \sim_{p} b, \quad w \sim_{p} u \quad \Longrightarrow \quad \bar{\nabla}_{\alpha}^{\text {bas }, \bar{g}}(\beta, w) \sim_{p} \nabla_{a}^{\text {bas }, g}(b, u) .
$$

Equations (3.4), (3.5) and (3.8) hence imply that

$$
\bar{\nabla}^{\text {bas }, \bar{g}}=\nabla^{\mathbb{V}} \oplus p^{!}\left(\nabla^{\text {bas }, g}\right) .
$$

Now form the affine connections

$$
\begin{array}{lll}
\nabla^{\mathrm{aff}}: & A \times T \Delta^{1} \curvearrowright \operatorname{Ad}(A) \times \Delta^{1}, & \nabla^{\mathrm{aff}}=t_{0} \nabla^{\mathrm{bas}}+t_{1} \nabla^{\mathrm{bas}, g}, \\
\bar{\nabla}^{\mathrm{aff}}: p^{!}(A) \times T \Delta^{1} \curvearrowright \operatorname{Ad}\left(p^{!}(A)\right) \times \Delta^{1}, & \bar{\nabla}^{\mathrm{aff}}=t_{0} \bar{\nabla}^{\text {bas }}+t_{1} \bar{\nabla}^{\text {bas }, \bar{g}}
\end{array}
$$

used respectively to compute $\operatorname{cs}\left(\nabla^{\text {bas }}, \nabla^{\text {bas }, g}\right)$ and $\operatorname{cs}\left(\bar{\nabla}^{\text {bas }}, \bar{\nabla}^{\text {bas }, \bar{g}}\right)$. Then equations (3.7) and (3.9) imply that

$$
\bar{\nabla}^{\mathrm{aff}}=\operatorname{pr}^{!}\left(\nabla^{\mathbb{V}}\right) \oplus\left(p, \mathrm{id}_{\Delta^{1}}\right)^{!}\left(\nabla^{\mathrm{aff}}\right),
$$

whence

$$
\operatorname{Tr}_{s}\left(R_{\bar{\nabla}^{\mathrm{aff}}}^{q}\right)=\operatorname{pr}^{*} \operatorname{Tr}_{s}\left(R_{\nabla^{\mathbb{V}}}^{q}\right)+\left(p, \mathrm{id}_{\Delta^{1}}\right)^{*} \operatorname{Tr}_{s}\left(R_{\nabla^{\mathrm{aff}}}^{q}\right)
$$

and so

$$
\begin{aligned}
\operatorname{cs}^{q}\left(\bar{\nabla}^{\text {bas }}, \bar{\nabla}^{\text {bas }, \bar{g}}\right) & =-f_{\Delta^{1}} \operatorname{Tr}_{s}\left(R_{\bar{\nabla}^{\text {aff }}}^{q}\right)=-f_{\Delta^{1}}\left(p, \mathrm{id}_{\Delta^{1}}\right)^{*} \operatorname{Tr}_{s}\left(R_{\nabla^{\text {aff }}}^{q}\right) \\
& =-p^{*} f_{\Delta^{1}} \operatorname{Tr}_{s}\left(R_{\nabla^{\text {aff }}}^{q}\right)=p^{*} \operatorname{cs}\left(\nabla^{\text {bas }}, \nabla^{\text {bas }, g}\right) .
\end{aligned}
$$

This shows that (3.1) holds true, and concludes the proof that $\operatorname{char}\left(p^{!}(A)\right)=p^{*} \operatorname{char}(A)$.

\section{Acknowledgements}

Work partially supported by the Nederlandse Organisatie voor Wetenschappelijk Onderzoek (Vrije Competitie grant "Flexibility and Rigidity of Geometric Structures" 612.001.101) and by IMPA (CAPES-FORTAL project). I would like to thank Ioan Mărcuţ, Ori Yudilevich, Rui Loja Fernandes, Olivier Brahic and David Martínez-Torres. I am also grateful to the anonymous referees for their many useful comments. 


\section{References}

[1] Abad C.A., Crainic M., Representations up to homotopy of Lie algebroids, J. Reine Angew. Math. 663 (2012), 91-126, arXiv:0911.2859.

[2] Bursztyn H., Lima H., Meinrenken E., Splitting theorems for Poisson and related structures, J. Reine Angew. Math., to appear, arXiv:1605.05386.

[3] Caseiro R., Fernandes R.L., The modular class of a Poisson map, Ann. Inst. Fourier (Grenoble) 63 (2013), 1285-1329, arXiv:1103.4305.

[4] Crainic M., Chern characters via nonlinear connections, math.DG/0009229.

[5] Crainic M., Differentiable and algebroid cohomology, van Est isomorphisms, and characteristic classes, Comment. Math. Helv. 78 (2003), 681-721, math.DG/0008064.

[6] Crainic M., Fernandes R.L., Secondary characteristic classes of Lie algebroids, in Quantum Field Theory and Noncommutative Geometry, Lecture Notes in Phys., Vol. 662, Springer, Berlin, 2005, 157-176.

[7] Crainic M., Fernandes R.L., Martínez Torres D., Poisson manifolds of compact types (PMCT 1), J. Reine Angew. Math., to appear, arXiv:1510.07108.

[8] Damianou P.A., Fernandes R.L., Integrable hierarchies and the modular class, Ann. Inst. Fourier (Grenoble) 58 (2008), 107-137, math.DG/0607784.

[9] del Hoyo M., Ortiz C., Morita equivalences of vector bundles, Int. Math. Res. Not., to appear, arXiv:1612.09289.

[10] Evens S., Lu J.-H., Weinstein A., Transverse measures, the modular class and a cohomology pairing for Lie algebroids, Quart. J. Math. Oxford 50 (1999), 417-436, dg-ga/9610008.

[11] Fernandes R.L., Lie algebroids, holonomy and characteristic classes, Adv. Math. 170 (2002), 119-179, math.DG/0007132.

[12] Frejlich P., Mărcut I., The homology class of a Poisson transversal, Int. Math. Res. Not., to appear, arXiv:1704.04724.

[13] Ginzburg V.L., Grothendieck groups of Poisson vector bundles, J. Symplectic Geom. 1 (2001), 121-169, math.DG/0009124.

[14] Ginzburg V.L., Golubev A., Holonomy on Poisson manifolds and the modular class, Israel J. Math. 122 (2001), 221-242, math.DG/9812153.

[15] Ginzburg V.L., Lu J.-H., Poisson cohomology of Morita-equivalent Poisson manifolds, Int. Math. Res. Not. 1992 (1992), 199-205.

[16] Grabowski J., Modular classes of skew algebroid relations, Transform. Groups 17 (2012), 989-1010, arXiv:1108.2366.

[17] Gracia-Saz A., Mehta R.A., Lie algebroid structures on double vector bundles and representation theory of Lie algebroids, Adv. Math. 223 (2010), 1236-1275, arXiv:0810.0066.

[18] Guillemin V., Miranda E., Pires A.R., Codimension one symplectic foliations and regular Poisson structures, Bull. Braz. Math. Soc. (N.S.) 42 (2011), 607-623, arXiv:1009.1175.

[19] Huebschmann J., Duality for Lie-Rinehart algebras and the modular class, J. Reine Angew. Math. 510 (1999), 103-159, dg-ga/9702008.

[20] Kosmann-Schwarzbach Y., Modular vector fields and Batalin-Vilkovisky algebras, in Poisson Geometry (Warsaw, 1998), Banach Center Publ., Vol. 51, Polish Acad. Sci. Inst. Math., Warsaw, 2000, 109-129.

[21] Kosmann-Schwarzbach Y., Poisson manifolds, Lie algebroids, modular classes: a survey, SIGMA 4 (2008), 005, 30 pages, arXiv:0710.3098.

[22] Kosmann-Schwarzbach Y., Laurent-Gengoux C., Weinstein A., Modular classes of Lie algebroid morphisms, Transform. Groups 13 (2008), 727-755, arXiv:0712.3021.

[23] Kosmann-Schwarzbach Y., Weinstein A., Relative modular classes of Lie algebroids, C. R. Math. Acad. Sci. Paris 341 (2005), 509-514, math.DG/0508515.

[24] Koszul J.-L., Crochet de Schouten-Nijenhuis et cohomologie, Astérisque (1985), 257-271.

[25] Kubarski J., Fibre integral in regular Lie algebroids, in New Developments in Differential Geometry (Budapest 1996), Kluwer Acad. Publ., Dordrecht, 1999, 173-202.

[26] Kubarski J., The Weil algebra and the secondary characteristic homomorphism of regular Lie algebroids, in Lie Algebroids and Related Topics in Differential Geometry (Warsaw, 2000), Banach Center Publ., Vol. 54, Polish Acad. Sci. Inst. Math., Warsaw, 2001, 135-173. 
[27] Kubarski J., Mishchenko A., Nondegenerate cohomology pairing for transitive Lie algebroids, characterization, Cent. Eur. J. Math. 2 (2004), 663-707.

[28] Mehta R.A., Lie algebroid modules and representations up to homotopy, Indag. Math. (N.S.) 25 (2014), 1122-1134, arXiv:1107.1539.

[29] Stiénon M., Xu P., Modular classes of Loday algebroids, C. R. Math. Acad. Sci. Paris 346 (2008), 193-198, arXiv:0803.2047.

[30] Vaisman I., Characteristic classes of Lie algebroid morphisms, Differential Geom. Appl. 28 (2010), 635-647, arXiv:0812.4658.

[31] Weinstein A., The modular automorphism group of a Poisson manifold, J. Geom. Phys. 23 (1997), 379-394.

[32] Xu P., Gerstenhaber algebras and BV-algebras in Poisson geometry, Comm. Math. Phys. 200 (1999), 545560, dg-ga/9703001. 